\title{
Simultaneous bilateral stereotactic procedure for deep brain stimulation implants: a significant step for reducing operation time
}

\author{
Erich Talamoni Fonoff, MD, PhD, ${ }^{1,2}$ Angelo Azevedo, MD, ${ }^{1}$ Jairo Silva dos Angelos, MD, ${ }^{1}$ \\ Raquel Chacon Ruiz Martinez, PhD, ${ }^{2}$ Jessie Navarro, MD, ${ }^{1}$ Paul Rodrigo Reis, BS, ${ }^{1}$ \\ Miguel Ernesto San Martin Sepulveda, MD, ${ }^{1}$ Rubens Gisbert Cury, MD, PhD, ${ }^{1}$ \\ Maria Gabriela dos Santos Ghilardi, MD, ${ }^{1}$ Manoel Jacobsen Teixeira, MD, PhD, ${ }^{1}$ and \\ William Omar Contreras Lopez, MD, PhD' ${ }^{1}$
}

\begin{abstract}
1Division of Functional Neurosurgery of Institute of Psychiatry, Department of Neurology, University of São Paulo Medical School; and 'Laboratory of Neuromodulation, Institute of Teaching and Research, Hospital Sirio-Libanês, São Paulo, Brazil
\end{abstract}

OBJECTIVE Currently, bilateral procedures involve 2 sequential implants in each of the hemispheres. The present report demonstrates the feasibility of simultaneous bilateral procedures during the implantation of deep brain stimulation (DBS) leads.

METHODS Fifty-seven patients with movement disorders underwent bilateral DBS implantation in the same study period. The authors compared the time required for the surgical implantation of deep brain electrodes in 2 randomly assigned groups. One group of 28 patients underwent traditional sequential electrode implantation, and the other 29 patients underwent simultaneous bilateral implantation. Clinical outcomes of the patients with Parkinson's disease (PD) who had undergone DBS implantation of the subthalamic nucleus using either of the 2 techniques were compared.

RESULTS Overall, a reduction of $38.51 \%$ in total operating time for the simultaneous bilateral group (136.4 \pm 20.93 minutes) as compared with that for the traditional consecutive approach ( $220.3 \pm 27.58$ minutes) was observed. Regarding clinical outcomes in the PD patients who underwent subthalamic nucleus DBS implantation, comparing the preoperative off-medication condition with the off-medication/on-stimulation condition 1 year after the surgery in both procedure groups, there was a mean $47.8 \% \pm 9.5 \%$ improvement in the Unified Parkinson's Disease Rating Scale Part III (UPDRS-III) score in the simultaneous group, while the sequential group experienced $47.5 \% \pm 15.8 \%$ improvement $(p=$ 0.96). Moreover, a marked reduction in the levodopa-equivalent dose from preoperatively to postoperatively was similar in these 2 groups. The simultaneous bilateral procedure presented major advantages over the traditional sequential approach, with a shorter total operating time.

CONCLUSIONS A simultaneous stereotactic approach significantly reduces the operation time in bilateral DBS procedures, resulting in decreased microrecording time, contributing to the optimization of functional stereotactic procedures. http://thejns.org/doi/abs/10.3171/2015.7.JNS151026

KEY WORDS deep brain stimulation; movement disorders; Parkinson disease; microelectrode recording; brain shift; functional neurosurgery

$\mathrm{B}$ ILATERAL deep brain stimulation (DBS) has become one of the most important neurosurgical techniques for the treatment of motor $^{7,8}$ and nonmotor ${ }^{2}$ symptoms of Parkinson's disease (PD) and other movement disorders. ${ }^{1,5}$ In recent years, many efforts have been made to improve therapeutic safety, accuracy, and comfort for pa- tients treated via DBS. Currently, most centers perform bilateral implantation using the same operation. ${ }^{10}$ Standard procedures normally involve introducing 2 sequential unilateral hemispheric implants in 1 lengthy operation. Not infrequently, implants on the second side can be troublesome either because brain shift is more likely to occur dur-

ABBREVIATIONS DBS = deep brain stimulation; GPi = globus pallidus internus; PD = Parkinson's disease; STN = subthalamic nucleus; UPDRS-III = Unified Parkinson's Disease Rating Scale Part III; Vim = ventralis intermedius nucleus.

SUBMITTED May 6, 2015. ACCEPTED July 27, 2015.

INCLUDE WHEN CITING Published online December 18, 2015; DOI: 10.3171/2015.7.JNS151026. 
ing prolonged procedures or because patient collaboration fades after delayed hours on the operating table. ${ }^{9}$ As bilateral DBS is consolidated for movement disorders and is under investigation for the treatment of various neuropsychiatric diseases, technical improvements are welcome. Thus, we present the results of performing simultaneous bilateral implantation procedures, demonstrating its feasibility and impact on shortening the time required for the surgery compared with that needed for the traditional sequential bilateral approach.

\section{Methods}

Our local ethics committee approved this study. The procedures were performed within the period from June 2012 to December 2014. It was an open study that primarily compared the operating time required for the cranial portion of the bilateral DBS implantation procedure. After giving informed consent, 57 patients with movement disorders - mostly PD but also dystonia and tremor-underwent bilateral DBS implantation in the same operation in the globus pallidus internus (GPi), the subthalamic nucleus (STN), or the thalamic ventralis intermedius nucleus (Vim), according to the medical indication. Simple electronic randomization was used to assign subjects to the 2 procedural groups, so 28 patients underwent conventional sequential bilateral DBS implantation (one side after the other), whereas the other 29 patients were subjected to the simultaneous bilateral approach.

Considering that most of the subjects were PD patients receiving bilateral implants in the STN (34 patients), we compared the clinical effects of STN stimulation between the groups undergoing each of the techniques by using the Unified Parkinson's Disease Rating Scale Part III (UPDRS-III), Hoehn and Yahr Scale, and levodopaequivalent dose before and 1 year after DBS. Only 20 patients who had completed 1-year follow-up were considered for this analysis.

\section{Operative Technique}

Permanent quadripolar DBS electrodes (model 3387, Medtronic Inc.) were implanted bilaterally in the GPi, the STN, or the Vim using stereotactic planning softwarebased image guidance (CT/MRI fusion/Mevis Neurosurgery Planning Software, Mevis). The operative technique was performed after administering a local anesthetic and consisted of routine steps, as described below.

We used an isocentric stereotactic frame system (TM03 Micromar/BMS-125E Bramsys) with 2 half arcs attached to the same frame. As requested by us, the manufacturer modified the system from its original version to meet the required specifications for the simultaneous bilateral approach. Thus, both half arcs could be simultaneously mounted, avoiding collision, which would occur at the midline if they were symmetrically positioned. This system was used for both approaches, performed either sequentially, using $1 \mathrm{arc}$, or simultaneously, using 2 arcs (Fig. 1).

The procedures for preoperative MRI, frame fixation, and stereotomography were the same for all patients. Following a routine antiseptic procedure and local anesthetic

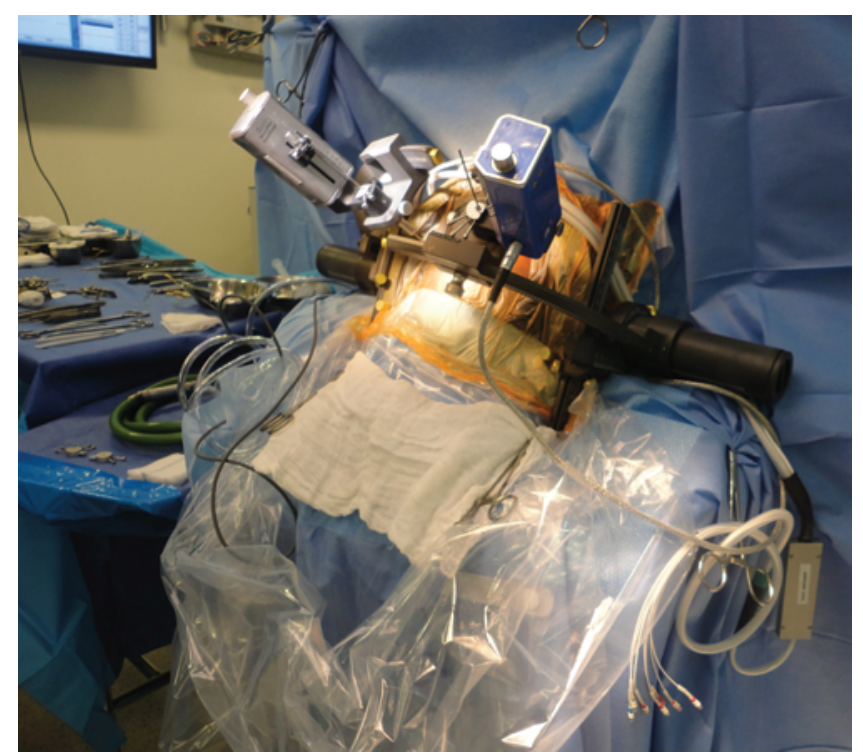

FIG. 1. Frame mounted with both half arcs for the simultaneous bilateral approach. Figure is available in color online only.

injection, the skin incision was marked and performed, and then bur holes were made (Step 1 in Fig. 2). From this step onward, the procedures consisted of either sequential (Fig. 2A) or simultaneous (Fig. 2B) microrecording and DBS electrode implantation (Steps 2 to 5 in Fig. 2). The bilateral system provided independent stereotactic coordinates for each of the hemispheres, and probe insertion was performed with 1 microdrive on each side in the simultaneous bilateral approach. In both types of procedure, the routine microelectrode recording technique was employed, using usually 2 microelectrodes for the GPi and 3 microelectrodes for recording in the STN on each side (Inomed Medizintechnik $\mathrm{GmbH}$ ). The simultaneous approach also allowed microelectrode recording to be performed simultaneously, with at least 2 channels on each side and recording channels converging to the same 5-channel amplifier. After the recordings, macrostimulation was performed for each of the hemispheres separately, eliciting motor effects on the contralateral side. Then, the microelectrodes were replaced with a permanent DBS electrode along the desired track, according to the clinical findings on both sides. As a routine procedure, following definitive electrode implantation, the position of each electrode was always verified using orthogonal radiographs. The electrodes were subsequently fixed to the skull, as a routine. ${ }^{3}$ Operative time was calculated from the time of the first incision to the moment of the last scalp stitch. Neither the time for frame fixation and preoperative tomography nor the operating time required for pulse generator implantation was computed in this study, as the same standard technique was employed for all patients.

To compare motor outcomes between the sequential and simultaneous groups we employed the Mann-Whitney unpaired t-test with significance set at $\mathrm{p}<0.05$.

\section{Results}

Demographic data are displayed in Table 1. Overall, 

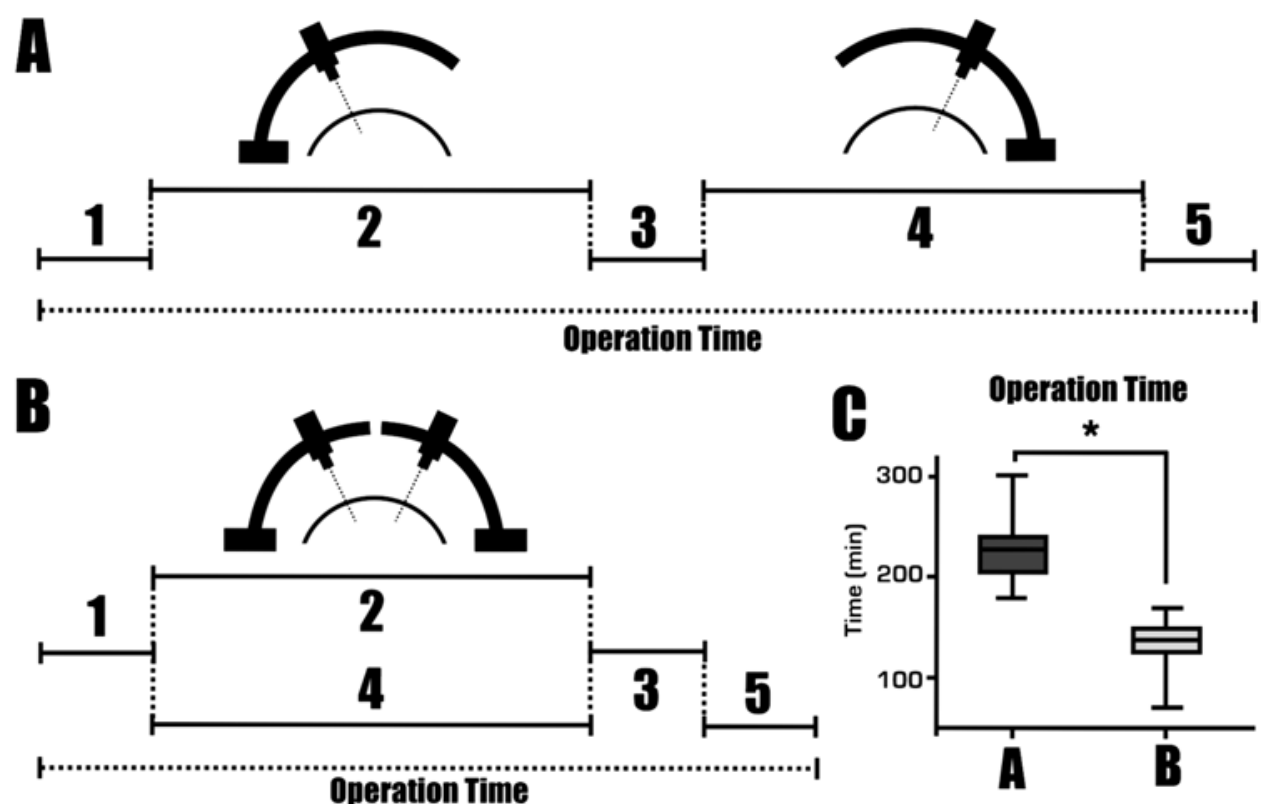

FIG. 2. Representation of each step of the surgical procedure: Step 1, skin incision and skull perforation; Steps 2 and 4, insertion of guide tubes and microelectrode recording electrodes, system connections, and actual recordings along descending trajectories, replacement of microelectrodes with the DBS electrode along the desired microelectrode track, followed by a fluoroscopy check; Steps 3 and 5 , clinical testing and electrode fixation and rechecking of electrode positioning if necessary. A: Timeline for sequential bilateral procedure. B: Timeline for simultaneous approach. C: Graph shows the significant difference between the time required for the consecutive traditional approach and the simultaneous bilateral approach: $220.3 \pm 27.58$ minutes $(A)$ and $136.4 \pm$ 20.93 minutes (B; Mann-Whitney unpaired t-test, $\mathrm{p}<0.0001)$. Asterisk indicates statistical significance.

the simultaneous bilateral implant procedures were 38.5\% faster than the conventional bilateral approach (Fig. 2C). The average surgical time for the simultaneous group was $136.4 \pm 20.93$ minutes, while the consecutive traditional approach took $220.3 \pm 27.58$ minutes (Mann-Whitney unpaired t-test, difference between means $-83.97 \pm 6.429$, $95 \%$ CI -96.84 to $-71.09, p<0.0001$ ). The proposed simultaneous approach resulted in the superimposition of a significant portion of the procedure, mainly regarding microelectrode recording, DBS electrode positioning, and the fluoroscopy check (Fig. 2B). The lateral fluoroscopy check was more difficult in the simultaneous procedure because overlap of the leads was frequent, requiring the surgeon to employ some strategy. We used either the variation in the electrode image size due to the parallax effect $^{6}$ (the image of the electrode that is closer to the fluoroscopy source appears larger than the more distant one), or a simple maneuver of retracting the inner radiopaque electrode stylet from one of the electrodes, leaving the electrode in place to distinguish the electrode on the right from that on the left. Macrostimulation and clinical test- ing were performed as routine procedures, one after the other, on the right or the left, depending on the choice of the neurophysiologist.

The 2 groups of PD patients who underwent STN implantation were considered objectively homogeneous in the preoperative state, and no significant differences in the clinical outcomes were observed between the groups 1 year after the DBS implant. Independent of the surgical technique (simultaneous or sequential approach), the patients had a mean improvement of approximately $47 \%$ in the motor score UPDRS-III, comparing the off-medication condition with the off-medication/on-stimulation condition 1 year after the procedure. Moreover, a marked reduction in the levodopa-equivalent dose from preoperatively to postoperatively was observed in these 2 groups (Table 2 ).

\section{Discussion}

This study demonstrates the feasibility of simultaneous bilateral procedures for the implantation of DBS leads. In addition to the modifications made to the stereotactic sys-

TABLE 1. Summary of characteristics in 57 patients who underwent DBS implantation

\begin{tabular}{|c|c|c|c|c|c|}
\hline Parameter & \multicolumn{2}{|c|}{ Simultaneous Approach } & \multicolumn{3}{|c|}{ Sequential Approach } \\
\hline Age in yrs & \multicolumn{2}{|c|}{$52.2 \pm 8.9$} & \multicolumn{3}{|c|}{$55 \pm 17$} \\
\hline Sex & \multicolumn{2}{|c|}{$19 \mathrm{M}, 10 \mathrm{~F}$} & \multicolumn{3}{|c|}{$16 \mathrm{M}, 12 \mathrm{~F}$} \\
\hline Diagnosis/DBS indication & $26 \mathrm{PD}$ & 3 dystonia & $17 \mathrm{PD}$ & 1 tremor & 10 dystonia \\
\hline Target of implant & 18 STN & $11 \mathrm{GPi}$ & 16 STN & $1 \mathrm{Vim}$ & $11 \mathrm{GPi}$ \\
\hline Op time in mins & $138.8 \pm 17.2$ & $135 \pm 20.92$ & $223.3 \pm 26.16$ & 210 & $221 \pm 27.22$ \\
\hline
\end{tabular}


TABLE 2. Motor outcome data in PD patients who received STN DBS implants via the simultaneous or sequential approach

\begin{tabular}{lccc}
\hline \multicolumn{1}{c}{ Parameter } & Simultaneous Approach & Sequential Approach & p Value* $^{*}$ \\
\hline Mean age in yrs & $50.3 \pm 9.5$ & $56 \pm 9.5$ & 0.26 \\
\hline Sex & $8 \mathrm{M}, 2 \mathrm{~F}$ & $7 \mathrm{M}, 3 \mathrm{~F}$ & 0.44 \\
\hline Mean disease duration in yrs & $10.2 \pm 3.8$ & $14.6 \pm 5.8$ & 0.17 \\
\hline Mean UPDRS-III score in preop off-med condition & $44.4 \pm 6.7$ & $41.6 \pm 11.9$ & 0.54 \\
\hline Mean UPDRS-III score in off-med/on-stim condition & $23.5 \pm 6.7$ & $21.7 \pm 10.1$ & 0.70 \\
\hline Mean preop Hoehn \& Yahr score & $2.8 \pm 0.8$ & $2.8 \pm 0.7$ & 1.00 \\
\hline Mean postop Hoehn \& Yahr score & $2.65 \pm 0.5$ & $2.2 \pm 10.4$ & 0.12 \\
\hline Mean preop LED & $1373.3 \pm 277.0$ & $1188.2 \pm 425.5$ & 0.42 \\
\hline Mean postop LED & $697.5 \pm 367.8$ & $620.0 \pm 232.6$ & 0.65 \\
\hline Mean UPDRS-III \% improvement & $47.8 \pm 9.5$ & $47.5 \pm 15.8$ & 0.96 \\
\hline
\end{tabular}

LED = levodopa-equivalent dose; off-med = patients with no effects of dopaminergic medication; on-stim = patients under the effects of therapeutic stimulation in the subthalamic nucleus.

* Mann-Whitney unpaired t-test was used to compare data in the 2 groups. Significance at $p<0.05$.

tem, the new approach required some changes to the routine surgical steps. The surgical team had to focus more attention while setting up the stereotactic coordinates on each side and to double check the coordinates to avoid deliberate mistakes. Additionally, a great deal of attention was required from the surgeon during the fluoroscopic check of electrode positioning because the electrode's images were frequently totally overlapping. However, the new procedure was not considered more difficult or less convenient by the team, which is already used to similar but longer procedures. Overall, the surgeons and the neurophysiologist felt that the procedures were uneventful and that the patients tolerated the simultaneous approach more easily and responded to clinical testing as satisfactorily as in sequential approach.

This is the first comparative study showing the substantial impact of a bilateral stereotactic system for simultaneous DBS electrode implantation. We observed significant shortening of the time required for the procedure-close to $40 \%$. Overall, shorter procedures are more comfortable for the patient, who is therefore more likely to cooperate with the clinical examination, possibly improving the information provided to the surgical team during the process of decision making regarding electrode positioning. Notably, the simultaneous procedure offers direct access to the target by allowing the surgeon to insert the guide tubes on both sides immediately after the dural opening. Even if a significant amount of air penetrates the intracranial cavity, causing brain dislocation, the guide tubes are already in place, holding the brain around the target to some degree. Thus, both sides are subjected to the operation under the same conditions as the first side in traditional procedures. Although this idea is fairly intuitive for surgeons with practice in this kind of procedure, it remains theoretical because this study was not initially designed to test this hypothesis. So, whether this technique reduces the risk of brain shift by shortening the total time of bur hole exposure is still a matter of speculation. No significant brain shift was observed in either of the study groups possibly because the surgeon adopted a technique of sealing the bur hole immediately after probe insertion as a routine.
Even though the simultaneous technique used in this study includes microrecording, which usually increases the time required for the procedure, the approach can also be applied in procedures without microrecording, perhaps shortening them as well. Additionally, simultaneous microrecording is possible with this technique, which could enhance our understanding of the neurophysiology of interhemispheric cross-talk during movement control, as suggested by Franzini et al. ${ }^{4}$

Although cost calculations depend on the type of health system in place in each country, shorter procedures may be less expensive than longer ones, which could be considered an additional advantage of the simultaneous bilateral procedures. However, if multiple microrecording electrodes are required, depending on the technique applied, the cost may be increased given doubling in the number of electrodes required in simultaneous procedures.

\section{Conclusions}

In summary, the simultaneous bilateral implantation procedure was significantly faster and better tolerated by patients than the traditional approach. Moreover, the PD patients who underwent the simultaneous approach for implantation of bilateral STN DBS experienced the same motor benefits as the group that underwent the sequential approach. Although not evaluated in this study, there is a theoretical possibility that the simultaneous approach may decrease the risk of brain shifting and its deleterious effects during intraoperative target determination and realtime, simultaneous recording of bilateral neuronal activity.

\section{Acknowledgments}

We thank all the research assistants and staff of Hospital SirioLibanês and Hospital das Clinicas HCFMUSP.

This work was supported by PROADI-SUS and a grant (FAPESP \#11/08575-7) from the government of São Paulo, Brazil (R.C.R.M.).

\section{References}

1. Brüggemann N, Kühn A, Schneider SA, Kamm C, Wolters 
A, Krause P, et al: Short- and long-term outcome of chronic pallidal neurostimulation in monogenic isolated dystonia. Neurology 84:895-903, 2015

2. Cury RG, Galhardoni R, Fonoff ET, Dos Santos Ghilardi MG, Fonoff F, Arnaut D, et al: Effects of deep brain stimulation on pain and other nonmotor symptoms in Parkinson disease. Neurology 83:1403-1409, 2014

3. Fonoff ET, Teixeira MJ, Nascimento CNG, Lopez WO: Aesthetically optimal deep brain stimulation technique in patients with alopecia. Neurocirugia (Astur) 26:206-208, 2015

4. Franzini A, Messina G, Rizzi M, Cordella R, Mazzone P: Bilateral simultaneous implant of electrodes within the subthalamic nucleus. Feasibility and advantages. Acta Neurochir (Wien) 155:1675-1676, 2013

5. Krack P, Hariz MI, Baunez C, Guridi J, Obeso JA: Deep brain stimulation: from neurology to psychiatry? Trends Neurosci 33:474-484, 2010

6. Mannila T: On parallax and variations in the stereoscopic image; with a view to stereofluoroscopy. Acta Radiol Suppl (139): 1-107, 1956

7. Moro E, Lozano AM, Pollak P, Agid Y, Rehncrona S, Volkmann J, et al: Long-term results of a multicenter study on subthalamic and pallidal stimulation in Parkinson's disease. Mov Disord 25:578-586, 2010

8. Odekerken VJJ, van Laar T, Staal MJ, Mosch A, Hoffmann CFE, Nijssen PCG, et al: Subthalamic nucleus versus globus pallidus bilateral deep brain stimulation for advanced Parkinson's disease (NSTAPS study): a randomised controlled trial. Lancet Neurol 12:37-44, 2013

9. Petersen EA, Holl EM, Martinez-Torres I, Foltynie T, Limousin P, Hariz MI, et al: Minimizing brain shift in stereo- tactic functional neurosurgery. Neurosurgery 67 (3 Suppl Operative):ons 213-ons221, 2010

10. Zrinzo L, Yoshida F, Hariz MI, Thornton J, Foltynie T, Yousry TA, et al: Clinical safety of brain magnetic resonance imaging with implanted deep brain stimulation hardware: large case series and review of the literature. World Neurosurg 76:164-172, 69-73, 2011

\section{Disclosures}

The authors report no conflict of interest concerning the materials or methods used in this study or the findings specified in this paper.

\section{Author Contributions}

Conception and design: Fonoff. Acquisition of data: Fonoff, Azevedo, dos Angelos, Martinez, Navarro, Reis, Sepulveda, Cury, Ghilardi, Contreras Lopez. Analysis and interpretation of data: Fonoff, Azevedo, dos Angelos, Martinez, Navarro, Cury, Ghilardi, Contreras Lopez. Drafting the article: Fonoff, Azevedo, dos Angelos, Martinez, Reis, Sepulveda, Cury, Ghilardi, Contreras Lopez. Critically revising the article: Fonoff, Martinez, Cury, Ghilardi. Reviewed submitted version of manuscript: Fonoff, Martinez, Navarro, Cury, Teixeira, Contreras Lopez. Approved the final version of the manuscript on behalf of all authors: Fonoff. Statistical analysis: Fonoff. Administrative/technical/ material support: Navarro, Teixeira. Study supervision: Teixeira.

\section{Correspondence}

Erich Talamoni Fonoff, Rua Dr. Ovídio Pires de Campos, 785, São Paulo, SP 01060-970, Brazil. email: fonoffet@usp.br. 